\title{
Clinical Factors in Successful Transfer of Cryopreserved Thawed Embryos
}

\author{
Nobumasa Kikuchi, ${ }^{1}$ Takanori Kamijo, ${ }^{1}$ Toshihiro Sekine, ${ }^{1}$ \\ Megumi Tsuchiya, ${ }^{1}$ Ibuki Yuji, ${ }^{1}$ Junro Kobayashi, ${ }^{1}$ \\ Masahiro Itoh, ${ }^{1}$ and Takashi Minegishi ${ }^{1}$
}

\begin{abstract}
Background \& Aims : To evaluate the effects of cryopreservation and setting an eye on changes in the blood Estradiol $\left(\mathrm{E}_{2}\right)$, Progesterone $\left(\mathrm{P}_{4}\right)$ levels and thickness of the endometrium before and after the transfer.

Methods : We studies whether these parameters can give us a clue to the mechanism leading to implantation and pregnancy from January 1999 to December 2000, 269 patients underwent 860 thawed embryo transfers.

Results and conclisions : 57 out of 269 cases achieved pregnancy, the pregnant rate being $21.2 \%$. Abortion occurred in 23 cases, the abortion rate being $40.3 \%$. No significant difference was found in mean age, mean number of embryo transfer, total score of embryo transfer in the Veeck's classification and mean score of embryo transfer between the pregnant and non pregnant cases. Pregnant cases tended to have many embryos of high grade transferred. No significant difference was found in blood $\mathrm{E}_{2}, \mathrm{P}_{4}$ levels and thickness of the endometrium in the pregnant and non pregnant cases.

There was no difference in mean age, mean number of embryo transfer, total score of embryo transfer in the Veeck's classification, mean score of embryo transfer and number of grades between the pregnant continuation cases and abortion cases. The cases of abortion showed a relatively high blood $\mathrm{E}_{2}$, level compared with the cases of clinically continued pregnancy. No difference in the blood $\mathrm{P}_{4}$ level and thickness of the endometrium was found between the two. The changes in these parameters can be used a guide in predicting prognosis of the pregnancy. (Kitakanto Med J $2002 ; 52: 117 \sim 121$ )
\end{abstract}

Key Words: $\mathrm{E}_{2}, \mathrm{P}_{4}$, thickness of endometrium

\section{Introduction}

Human cryopresered embryo transfer has become an indispensable technique at the hospital where in vitro fertilization (IVF) -embryo transfer (ET) is performed since Trounson et al reported first case of pregnancy/childbirth resulting from cryopresered embryo transfer. ${ }^{1}$

As a prerequisite to performing cryopresered thawed embryo transfer, it is necessary to synchronize the developmental stage of the endometrium and embryo for establishing pregnancy. Various factors are supposed to be concerned with this process, including the ability of pre-implantation embryo, adhesion of embryo to the endometrium, increased vascular permeation in the endometrial interstice, localized deciduation of membrane around the implanted embryo, infiltration of trophoblast and regulatory effect of ovarian steroid, pregnancy maintaining mechanism of the body. ${ }^{2-5}$

Setting an eye on changes in the blood Estradiol $\left(\mathrm{E}_{2}\right)$, Progesterone $\left(\mathrm{P}_{4}\right)$ levels and thickness of the endometrium before and after the transfer, therefore we studies whether these parameters can give us a clue to the mechanism leading to implantation and pregnancy. This paper is a report of the results of our study.

1 Department of Obstet \& Gynecol, Gunma University School of Medicine, 3-39-22 Showa- machi, Maebashi, Gunma 371-8511 Received : January 17, 2002

Address : NOBUMANA KIKUCHI Department of Obstet \& Gynecol, Gunma University School of Medicine, 3-39-22 Showa- machi, Maebashi, Gunma 371-8511 


\section{Materials and Methods}

The subjects consisted of 269 patients who had their fertilized ova frozen after IVF-ET at the Department of Obstetrics and Gynecology Gunma University School of Medicine, as outpatients for evaluation and treatment by assisted reproductive technologies between January 1999 and December 2000. In this study, the women were between 23 and 36 (mean 30) years of age. For pregnancy to occur as a prerequisite to performing cryopresered thawed embryo transfer, we use a method whereby the implantation environment isimproved by hormone replacement therapy. ${ }^{5-6}$ Cryopresered thawed embryo transfer with hormone replacement therapy was started using recipients with the anovulatory cycle or irregular ovulatory cycle in oocyte donation as the subjects. It has now become the main current in America and Europe. This method requires no LH surge for synchronization in the natural cycle, no monitoring of ovarian follicle by ultrasonic tomography and no determination of blood hormone levels, having advantages such as being able to set the date of embryo transfer easily and to improve the shape and thickness of the endometrium against diminished fertility of the endometrium with aging.

In cryopresered thawed embryo transfer with hormone replacement therapy practiced at our department, administration of gonadotropin releasing hormone analogue $(\mathrm{GnRHa}) 900 \mathrm{~g} /$ day is started from the middle of the luteal phase, and administration of Premarin (Serono Japan, Tokyo, Japan), a conjugated estrogenic hormone preparation is started after down regulation has been obtained (Fig 1). The dose of Premarin is increased every 4 days after inception of administration, and a combinational use of progesterone is started from day 13. On days 14 the embryo is thawed and the growth is confirmed by 1-day additional incubation. On the 15 transfer is performed, and thereafter Premarin and progesterone are administered continuously up to judgement on the occurrence of pregnancy two weeks later. In the case of the pronuclear phase, thawing and embryo transfer are

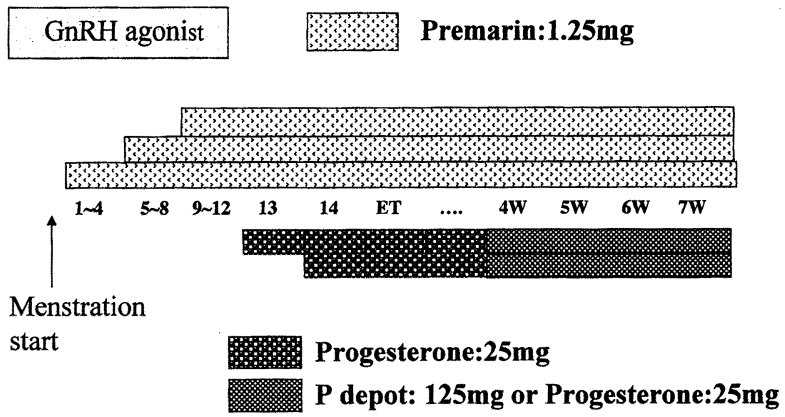

Fig 1 Cryopresered thawed embryo transfer with hormone replacement protocol at our department

performed on days 13 and 14 . Where pregnancy has been confirmed, the drugs are used up to around the 12 , th week when fetal and placental hormones are stabilized. As to progesterone, it is changed to a depot preparation at a time when fetal heartbeat has been confirmed. Blood is collected at the time of the dose being increased every 4 days after inception of Premarin administration, before and after embryo transfer and days 5 and 10 after embryo transfer. For the thickness of the endometrium, the maximum horizontal diameter of the structural halo was measured on the hypoechoic line outside the endometrial image confirmed by transvaginal ultrasonography. ${ }^{7}$ Morphology of the embryo was evaluated by scoring like G1-10points, G2-8 points in order of higher grades using the Veeck's classification. ${ }^{8}$ For statistical analysis, the Fisher's test was performed, and theststistical significance was defined as $\mathrm{P}(0.05)$.

\section{Results}

Changes in blood $\mathrm{E}_{2}, \mathrm{P}_{4}$ levels, thickness of endometrium and evaluation of embryos in cryopresered thawed embryo transfer.

Table 1 gives the breakdown of the pregnant and non pregnant cases before and after cryopresered thawed embryo transfer. In the present study, 57 out of 269 cases achieved pregnancy, the pregnant rate being 21.2\%. Abortion occurred in 23 cases, the abortion rate being $40.3 \%$. No significant difference

Table 1 The outcome of cryopresered thawed ET

\begin{tabular}{lcc}
\hline & Pregnant Case & Non Pregnant Case \\
\hline Mean of Age & $33.5 \pm 0.5$ & $34.1 \pm 0.3$ \\
\hline Patient cycles & 57 & 212 \\
\hline Mean number of ET & $3.7 \pm 0.1$ & $3.4 \pm 0.1$ \\
\hline Total score of ET in the Veeck's classification & $25.1 \pm 1.0$ & $23.7 \pm 0.6$ \\
\hline Mean score of ET in the Veeck's classification & $7.3 \pm 0.2$ & $7.2 \pm 0.1$ \\
\hline Mean number of ET High grade transferred (G1-G2) & $2.3 \pm 0.2^{*}$ & $1.6 \pm 0.2$ \\
\hline ET = embryo transfer & & \\
Veeck's classification score (G1-10 point, G2-8, G3-6, G4-4, G5-2) & \\
$* P(0.05)$.
\end{tabular}




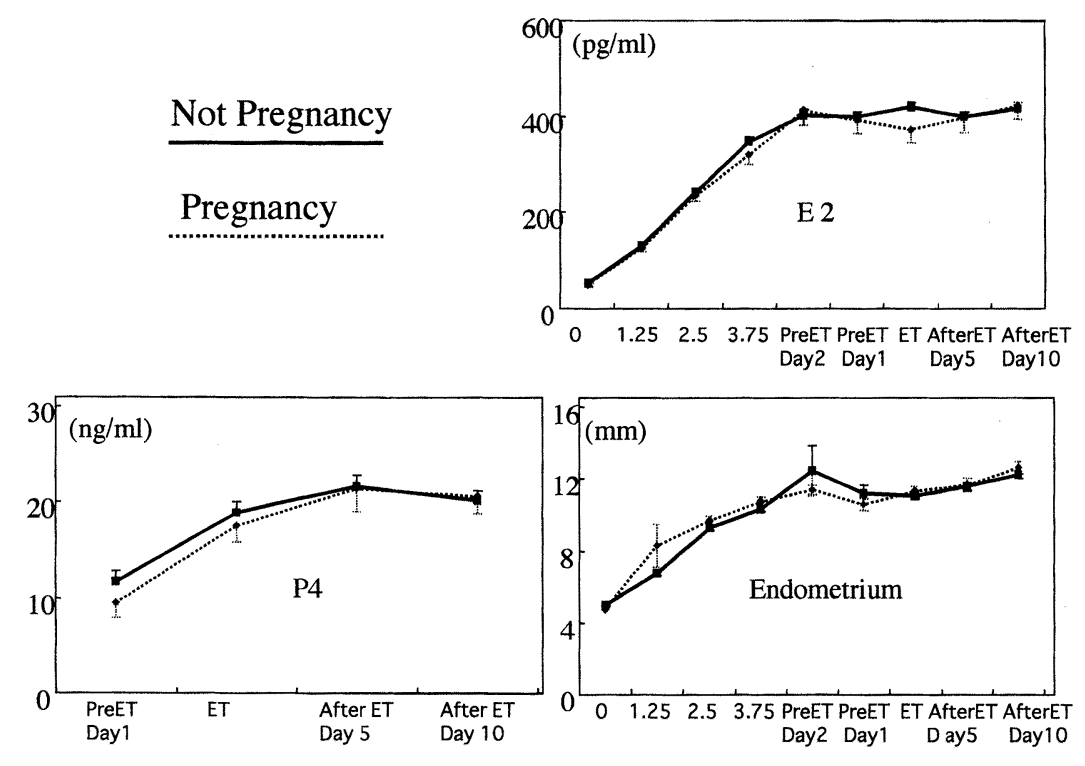

Fig 2 Changes in blood $\mathrm{E}_{2}, \mathrm{P}_{4}$ levels, thickness of endometrium in the pregnant and non pregnant cases

Table 2 The outcome of cryopresered thawed ET

\begin{tabular}{lcc}
\hline & Clinical Pregnant Case & Abortion Case \\
\hline Mean of Age & $33.6 \pm 0.6$ & $33.3 \pm 0.8$ \\
\hline Patient cycles & 34 & 23 \\
\hline Mean number of ET & $3.5 \pm 0.1$ & $3.4 \pm 0.2$ \\
\hline Total score of ET in the Veeck's classification & $24.9 \pm 1.3$ & $25.3 \pm 1.7$ \\
\hline Mean score of ET in the Veeck's classification & $7.1 \pm 0.3$ & $7.4 \pm 0.3$ \\
\hline Mean number of ET High grade transferred (G1-G2) & $2.2 \pm 0.2$ & $2.4 \pm 0.2$ \\
\hline ET=embryo transfer & & \\
Veeck's classification score (G1-10 point, G2-8, G3-6, G4-4, G5-2) & \\
*P $(0.05)$.
\end{tabular}

was found in mean age, mean number of embryo transfer, total score of embryo transfer in the Veeck's classification and mean score of embryo transfer between the pregnant and non pregnant cases. However, pregnant cases tended to have many embryos of high grade transferred.

Figure 2 illustrates changes in blood $\mathrm{E}_{2}, \mathrm{P}_{4}$ levels and thickness of the endometrium in the pregnant and non pregnant cases as well as the pregnant continuation cases and non continuation cases in cryopresered thawed embryo transfer with hormone replacement.

Table 2 gives the breakdown of the cases of clinically continued pregnancy and cases of abortion in 57 pregnant cases. There was no difference in mean age, mean number of embryo transfer, total score of embryo transfer in the Veeck's classification, mean score of embryo transfer and number of grades between the pregnant continuation cases and abortion cases. The cases of clinically continued pregnancy broke down to full term birth 31 cases, premature birth 3 cases, transvaginal delivery 20 cases and cesarean section 11 cases.

Figure 3 illustrates changes in blood $\mathrm{E}_{2}, \mathrm{P}_{4}$ levels and thickness of the endometrium in cases of clinically continued pregnancy and cases of abortion in thawed embryo transfer with hormone replacement. The cases of abortion showed a relatively high blood $\mathrm{E}_{2}$, level compared with the cases of clinically continued pregnancy. However, no difference in the blood $\mathrm{P}_{4}$ level and thickness of the endometrium was found between the two. The blood $\mathrm{E}_{2} / \mathrm{P}_{4}$ level tended to be high in the cases of abortion.

\section{Discussion}

The implantation rate of fertilized ova in cryopresered thawed embryo transfer is low at $10-15 \%$, there are many problems to be elucidated. ${ }^{5-6}$ As can be seen from the results of this study, blood $\mathrm{E}_{2}, \mathrm{P}_{4}$ levels showed no significant difference between the cases during the transfer cycle in both the pregnant cases and non pregnant cases. This fact may be taken as demonstrating that the method whereby $\mathrm{GnRHa}$ is used from the middle of the luteal phase, and ovarian steroid hormone is administered after obtaining down regulation to make the endometrium grow artificially is offering a fixed hormonal environment to all patients. ${ }^{9}$ 


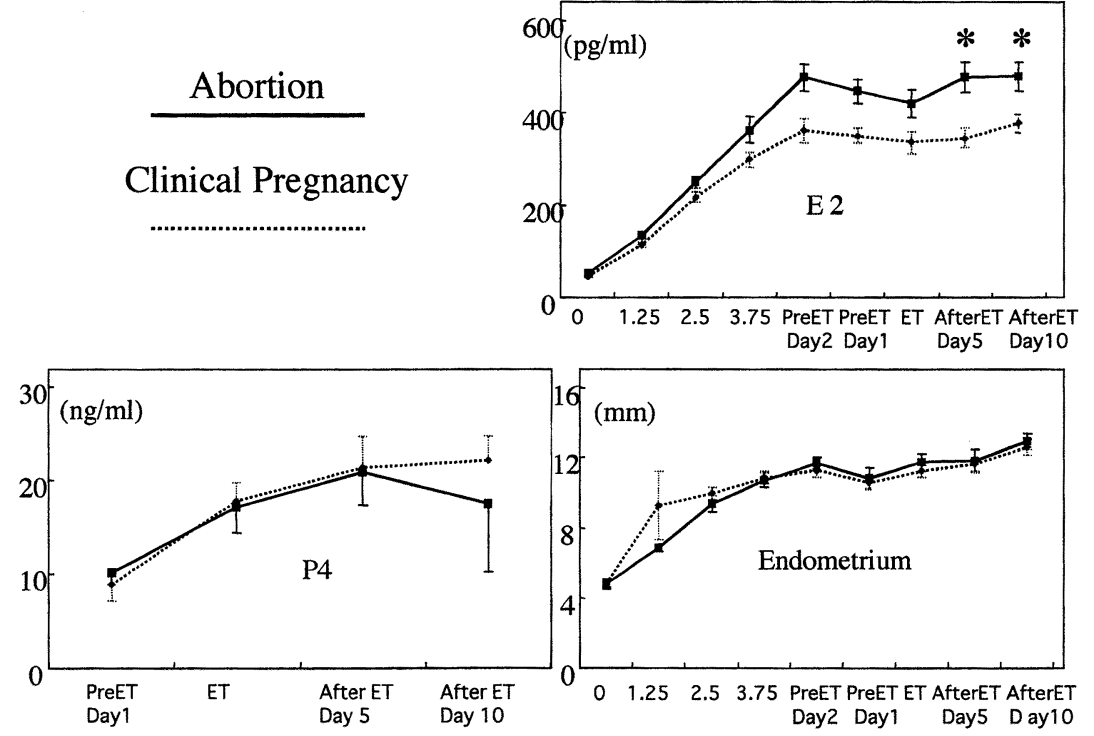

Fig 3 Changes in blood $\mathrm{E}_{2}, \mathrm{P}_{4}$ levels, thickness of endometrium in cases of clinical continued pregnancy and cases of abortion

However, the present study has produced results to indicate the difficulty of predicting what raises the pregnant rate and what method should be used to induce pregnancy merely from the behavior of hormones. Similar results were obtained from the thickness of the endometrium at about the same time, and no significant difference was found between the pregnant cases and non pregnant cases. How to transfer many good embryo of high grade on the Veeck's classification, a morphological method of evaluating embryo is the key to success in the pregnant cases. Similar reports have been published about cryopresercd thawed embryo transfer as well as fresh embryo transfer. ${ }^{10}$ The same is also true of the present study. The question of the quality of embryo can not be disregarded when the maternal age is taken into consideration.

When, however, only the pregnant cases were studied, the $\mathrm{E}_{2}$ level tended to be relatively high in the cases of abortion compared with the cases of clinically continued pregnancy. It is interesting to note that no difference in the grade of embryo, age and thickness of the endometrium was found and that blood $\mathrm{E}_{2} / \mathrm{P}_{4}$ levels were high. ${ }^{11}$ Implantation in humans starts when a blastula grown through fertilization and early development is adhered to the endometrium and ends when the entire blastula is completely wrapped up by the endometrium. ${ }^{12-13}$ It refers to a series of process from days 6,7 to day 13 after fertilization. For the normal implantation process to proceed requires a blastula normal implantation capacity and the normal endometrium to accept implantation. The endometrium un the implantation phase is preparing for implantation under the influence of progesterone and estrogen. ${ }^{14-15}$ However, it is known that a high $\mathrm{E}_{2} / \mathrm{P}_{4}$ ratio due to excessive secretion of estrogen impedes implantation even when progesterone is normal. ${ }^{16}$ The endometrium primarily causes dilatation of the helicine artery and an increase in the blood flow of the endometrial vessel, which accelerates the thickening of the endometrium. ${ }^{17}$ Deciduation at the time of implantation of fertilized ova is reportedly accelerated under the influence of estrogen and progesterone receptor present in the endometrium, while at the same time adhering factors such as laminin and fibronectin as well as growth factors such as IGF- (and EGF are produced to induce implantation of fertilized ova. ${ }^{17-20}$ Thesis effects are expected to disturb the hormone balance and thereby impede the building up of the interrelation between the embryo and endometrium leading to the implantation phase.

It is difficult to judge whether or not a woman is pregnant by changes in blood $\mathrm{E}_{2}, \mathrm{P}_{4}$ levels and thickness of the endometrium after cryopresered thawed embryo transfer. In the pregnant cases, however, it was possible to predict whether pregnancy continues or ends up in abortion by changes of blood $\mathrm{E}_{2}$ levels and blood $\mathrm{E}_{2} / \mathrm{P}_{4}$ levels. So, the changes in these parameters can be used $\mathrm{s}$ a guide in predicting prognosis of the pregnancy during examination at the out-patient clinic.

\section{References}

1. Trounson A, Mohr L. Human pregnancy following cryopreservation, thawing and transfer of an eight-cell embryo. Nature 1983; 305: 707709.

2. Cross JC, Werb Z, Fisher SJ. Implantation and 
the placenta key pieces of the development puzzle. Science 1994; 266 : 1508-1518.

3. Ghosh D, Sengupta J. Recent development in endocrinology and paracrinology of blastocyst implantation in the primate. Hum Reprod 1998 ; 4 : 153-168.

4. Sueoka K, Shiokawa S, Miyazaki T, et al. Integrins and reproductive physiology expression and modulation in fertilization, embryogenesis, and implantation. Fertil Steril 1997; 67 : 799-811.

5. Veeck LL, Maloney $\mathrm{M}$, Amundson $\mathrm{C}$, et al. Significantly enhanced pregnancy rates per cycle through cryopreservation and thaw of pronuclear stage oocytes. Fertil Steril 1993; 59: 1202-1207.

6. Queenan J, Veeck LL, Muasher S, et al. Transfer of cryopreservation - thawed pre-embryos in a natural cycle or a programmed cycle with exogeous hormonal replacement yields similar pregnancy results. Fertil Steril 1994; 62: 545550.

7. Sharara FI, Lim J, McClamrock HD. Endometrial pattern on the day of oocyte retrieval is more predictive of implantation success than the pattern or thickness on the day of hCG administration. J Assist Reprod Genet 1999; 16: 523528.

8. Veeck LL. Atlas of the Human Oocyte and Early Conceptus, Vol. 2, Williams \&Wilkins Co, Baltimore, 1991.

9. Kikuchi N, Masahiro I, Takashi M, et al. Epididymal and testicular sperm for intracytoplasmic sperm injection in the treatment of azoospermia. Kitakanto med J 2001; 51 : 249-534.

10. Queenan J, Ramey J, Seltman H, et al. Transfer of cryopreservation - thawed pre-embryos in a cycle using exogeous steroids without prior gonadotropin-releasing hormone agonist suppression yields favourable pregnancy results. Hum Reprod 1997; 12 : 1176-1180.

11. Irianni $\mathrm{F}$, Veeck $\mathrm{L}$, Toner $\mathrm{J}$, et al. Influence of number of pre-embryos transferred, progesterone level and oestradiol / progesterone ratio at thaw on pregnancy results during replacement of cryopreserved pre-embryos in natural cycles. Hum Reprod 1992; 7: 797-800.

12. Simon $\mathrm{C}$, Dominguez $\mathrm{F}$, Remohi $\mathrm{J}$, et al. Embryo effects in human implantation: embryonic regulation of endometrial molecules in human implantation. Ann-N-Y-Acad-Sci 2001; 943: 1-16.

13. Watson A, Barcroft L. Regulation of blastocyst formation. Front Biosci 2001; 6: 207-730.

14. de Ziegler D, Fanchin R, de Moustier B, et al. The hormonal control of endometrial receptivity estrogen and progesterone. J Repro Immunol 1998 ; 39: 149-166.

15. Lelaidier C, de Ziegler D, Freitas $S$, et al. Endometrium preparation with exogenous estradiol and progesterone for the transfer of cryopreserved blastocysts. Fertil Steril 1995; 63: 919-921.

16. Saito H, Sato F, Hirayama T, et al . Factors promoting embryo implantatopn in vitro fertilization and embryo transfer. Horm Res 1992; 37 : 164-168.

17. Kierszenbaum A. Decidualization and implantation: embryo-uterine bioinformatics at work. Mol Reprod Dev 2001 ; 59 : 123-125.

18. Reddy K Meheriji P. Integrin cell adhesion molecules in endometrium of fertile and infertile women throughout menstrual cycle. Indian $\mathbf{J}$ Biol ; 37 : 323-331.

19. Bilalis D, Klentzeris L, Fleming S. Immunohistochemical localization of extracellular matrix proteins in luteal phase endometrium of fertile and infertile patients. Hum Reprod 1996; 11 : 2713-2718.

20. Lennard S, Gerstenberg C, Allen W, et al. Expreeion of epidermal growth factor and its receptor in equine placental tissues. J Reprod Fertil 1998; 112: 49-57. 